\section{Automated generation of the optimal performance trade-offs of integrated inductors}

\author{
R. González-Echevarría, R. Castro-López, E. Roca, F.V.
} Fernández, J. Sieiro, N. Vidal and J.M. López-Villegas

\begin{abstract}
In this paper, a new methodology for the automated generation of the optimal performance trade-offs of integrated inductors is presented. The methodology combines a multiobjective optimization algorithm with electromagnetic simulation to get highly accurate results. A set of sized inductors is obtained showing the best performance trade-offs for a given technology. Unlike reported approaches for inductor synthesis, performance trade-offs are generated offline, i.e., before any specific inductance or quality factor are required. The tight efficiency vs. accuracy trade-off of existing approaches is, in this way, avoided and performance evaluation via electromagnetic simulation becomes affordable.
\end{abstract}

Index Terms-Inductor synthesis, multi-objective optimization, performance trade-offs, design methodologies.

\section{INTRODUCTION}

O ne of the major technical challenges associated with the integration of radio frequency (RF) circuits is the design of on-chip inductors, used in RF integrated circuits (ICs) for input/output matching networks, passive filters, low noise amplifiers, oscillators, etc. In all of these circuits, inductors should be carefully designed or selected, since both circuit performances and area are extremely dependent on them. The main characteristics to be considered when designing integrated inductors are equivalent inductance $\left(L_{e q}\right)$, quality factor $(Q)$, self-resonance frequency $(S R F)$ and area. Most of these performances are conflicting, i.e., one cannot be improved without worsening other. Also, they are closely related to the inductor geometric parameters and some are frequency-dependent. Therefore, finding the inductor with the appropriate performance trade-off for a specific application is a complicated task. This task is even more complex since accurately evaluating the inductor performances requires long and computationally expensive electromagnetic (EM) simulations. This problem is especially important in automated RF circuit design methodologies, where EM simulation is rarely used due to the large number of inductors that has to be iteratively evaluated.

Reported approaches deal with the inductor sizing problem either (a) indirectly, by optimizing the performances of the RF circuits in which they are embedded [1]-[6]; or (b) directly, by optimizing inductor performances like $L_{e q}, Q, S R F$ and area [7]-[16]. All of them share a common goal: they are intended for what can be called online optimization, i.e., the inductors are designed for some given specifications at the moment they are needed during the design of an RF circuit (see

R. González-Echevarría, R. Castro-López, E. Roca and F.V. Fernández are with IMSE-CNM, CSIC and Universidad de Sevilla, Spain. e-mail: pacov@imse-cnm.csic.es. J. Sieiro, N. Vidal and J.M. López-Villegas are with Dept. of Electronics, University of Barcelona, Spain.

This work has been partially supported by the TEC2010-14825 and TEC2010-21484 projects, funded by the Spanish Ministry of Economy and Competitiveness and ERDF, by the P12-TIC-1481 project, funded by Junta de Andalucia, and by CSIC project PIE 201350E058.
Fig. 1(a)). This brings a critical efficiency vs. accuracy tradeoff. As accurate EM simulation takes too long to be embedded into an optimization loop, some kind of approximate analytical or surrogate-based model is used, sometimes with iterative improvement of the model accuracy as the optimization progresses [16], [17]. Reported approaches differ in the optimization technique, the kind of approximate model used for performance evaluation or how the performance evaluator is integrated with the optimization technique. Another common feature of most reported approaches is that they are intended for the design of a single inductor, e.g. the goal is to get a certain inductance and quality factor at a given frequency with minimum area occupation and some constraints on the self-resonance frequency. This implies that trade-offs between inductor performances are not actually pursued.

In this paper, a new RF design paradigm based on offline optimization of inductor performances is proposed. Instead of designing a specific inductor when it is needed, the set of inductors showing the best performance trade-offs for a given technology is generated by formulating a multi-objective optimization problem. Although, analytical or surrogate models could be used to evaluate inductor performances, they face very significant accuracy problems when wide regions of the design parameter space or frequency are considered. Therefore, multi-objective optimization based on these models may not correctly converge to the regions with best performance trade-offs. However, considering that no specific performance value is pursued in our proposed approach, the generation can be performed offline, i.e. much before some specific inductance or quality factor value are required for an RF circuit design problem (see Fig. 1(b)). This implies that the critical efficiency vs. accuracy trade-off of online optimization approaches disappears. Thus, considerably higher computation times are acceptable, and accurate EM simulation becomes an attractive option for performance evaluation in the multi-objective optimization algorithm. Nevertheless, efficiency enhancement techniques have been applied in this approach, e.g. parallelization of the optimization process,
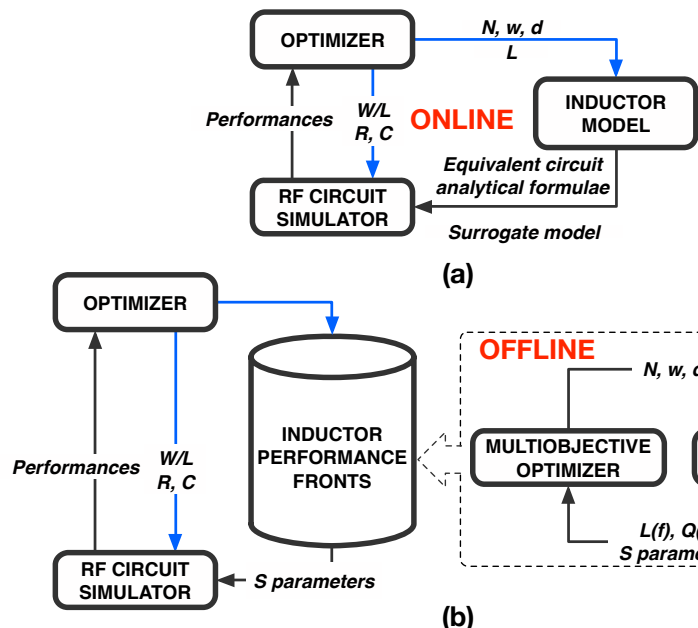

(a)

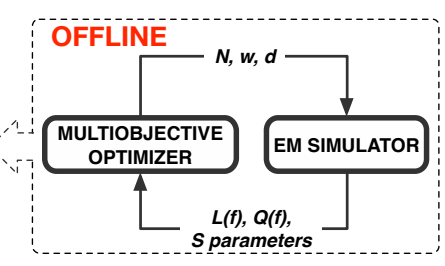

(b)

Fig. 1: RF circuit design flow with (a) online and (b) offline optimization of inductors. 
adaptive meshing and smart selection of sampling frequencies in the EM simulation, command line programming control of commercial tools and adaptive stopping criteria that will halt the optimization algorithm when no further improvement can be obtained.

The proposed approach can be used for more efficient RF circuit design. In addition, performance fronts are generated which enable easy exploration of trade-offs between the inductor performances.

\section{GENERATION OF THE PERFORMANCE TRADE-OFFS}

\section{A. Problem definition}

Finding the optimal trade-offs between the inductors performances (inductance and quality factor at one or more frequencies, and area) can be formulated as a multi-objective optimization problem, where two or more performances (objectives) are maximized or minimized, while some constraints are met. A solution to this problem is provided by populationbased multi-objective optimization evolutionary algorithms (MOEA). These algorithms are based on the evolution of a population of individuals (being each individual defined by a set of design variables) guided by the concept of Pareto dominance, which considers that given two solutions, a and $\mathbf{b}, \mathbf{a}$ is said to dominate $\mathbf{b}$ if all the objectives of $\mathbf{a}$ are not worse than those of $\mathbf{b}$ and there is at least one objective of $\mathbf{a}$ that is strictly better that the same objective of $\mathbf{b}$. In the same way, $\mathbf{a}$ is said to be a non-dominated solution if there is no other solution that dominates it. The result of the optimization is a set of non-dominated solutions showing the best trade-offs that can be obtained between the objectives, which is known as Pareto-optimal front (POF).

The MOEA NSGA-II [18] was selected as optimization algorithm in our approach, although its role could be replaced by any of the tens of multi-objective optimization algorithms reported in the literature.

In this work, the design variables are given by the number of turns, $N$, the inner diameter, $D_{i n}$, the width of the turns, $W$, and the spacing among turns, $S$, as illustrated in Fig.2. Constraints are imposed to ensure that the inductor behavior agrees with operating specifications (e.g., location of SRF with respect to operating frequency, maximum inductor area, etc.). The final POF obtained includes a set of inductors that represent the best trade-offs among those performances selected as objectives and that meet the constraints.
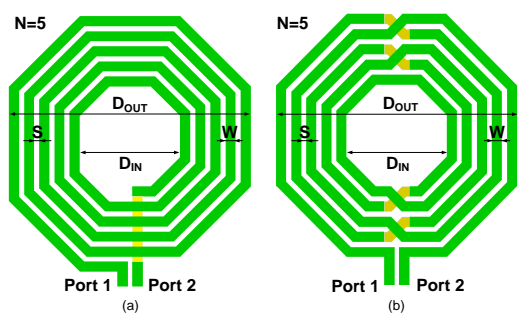

Fig. 2: Geometric parameters for (a) an octagonal asymmetric spiral inductor and (b) a symmetric inductor.

The performance evaluator used is an accurate EM simulator that provides the scattering parameters (S-parameters) of the two-port structure defined by an inductor. From these parameters, the equivalent input impedance, $Z_{e q}$, can be calculated and $L_{e q}$ and $Q$ can be obtained at any operating frequency, $f$. The self-resonance frequency, $S R F$, is defined as the frequency at which the imaginary part of $Z_{e q}$ is zero. The value of $Z_{e q}$ depends on the excitation conditions of the circuit where the inductor is included [19]. Usually, asymmetric inductors are driven by a non-differential excitation, whereas symmetric inductors are driven by a differential excitation. Therefore, each topology is evaluated accordingly to the equations reported in [20].

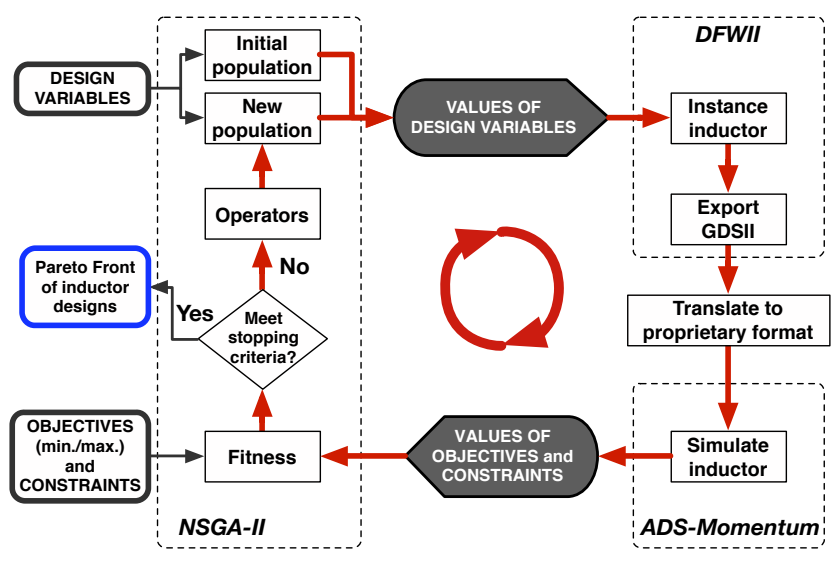

Fig. 3: Block diagram of the proposed optimization flow.

\section{B. Optimization flow}

The proposed flow is shown in Fig.3. The first step in this flow corresponds to the definition of input data: inductor topology, design variables and performance objectives and constraints. The range of possible values for each variable has to be also set. Although wide ranges of variables are generally allowed to enable a wide exploration of the design space, the technology process imposes a lower limit to metal widths in the inductor turns and a minimum size of the inner diameter. An upper limit can also be set from the desired maximum area of the inductors. A variation grid is also needed, which changes for each type of variable, due to technology process limitations (e.g., the minimum grid for turn widths is limited by the minimum change of metal widths allowed) or layout restrictions (e.g., the layout template used to generate the inductors may impose that the number of turns must be an integer number, or change in quarters of a turn only).

The flow continues by randomly generating the initial population: a population of individuals whose design variables have been set randomly within the ranges defined above. In order to evaluate the individuals of this and subsequent populations, the layout of each inductor (according to the values of its design variables) has to be first generated automatically. Each inductor of the population is electromagnetically simulated, obtaining the S-parameter matrix, which is used to calculate the values for inductance and quality factor at any desired frequencies. Postprocessing of these data provides the desired objective and constraint values. This process is repeated for all the inductors of the population. The results are fed back to the optimization algorithm. Once evaluated, individuals of this 
population are merged with the population of the previous generation. Then individuals of the merged population are ranked and the best ones are selected to conform the population of the next generation. If this population meets some stopping criteria, the optimization process halts. The non-dominated set of inductors (the Pareto-optimal front) represents the best trade-offs among the inductor performances. If the stopping criteria are not fulfilled, the evolutionary optimization algorithm creates a new population of inductors by applying different operators, like crossover and mutation, to the previous population. The complete process is then repeated. The most relevant parts of this flow are discussed in detail in the following sections.

\section{Automatic layout generation}

To evaluate the performances of the individuals of the population, the layout of each inductor has to be first generated according to the values of its design variables. Parameterized cells are used to instance the inductor layout in Cadence's Virtuoso platform for the corresponding inductor topology, exported into GDSII format, and automatically converted into an EM simulator-compatible layout format. The entire task is controlled in command-line programming to increase efficiency. A library of parameterized inductors cells with five different inductors topologies has been implemented: symmetric square with bridges at $90^{\circ}$ and at $45^{\circ}$, symmetric octagonal, spiral square and spiral octagonal.

\section{Performance evaluation}

The EM simulator Momentum has been used in our implementation as performance evaluator. Before any EM simulation is started, Green functions associated with the substrate are pre-computed. Although computationally expensive, once calculated for a certain frequency range, the information can be reused for any simulation within that frequency range.

Several parameters have to be set before starting the simulation: location of ports, the type of ports (that determine the simulation type), layers and substrate definition, the precomputed substrate files, simulation frequencies and mesh configuration. During the mesh definition, the inductor metal strips are divided by creating a grid pattern of cells of a certain size, depending, among other parameters, on the simulation frequency and width and length of the metal lines. The simulation time grows with the number of cells. Therefore, this number should be the minimum required to get the right accuracy. The generated mesh is specific to each inductor layout and it is generated before each simulation. In this methodology, the width of the outer mesh cells is changed according to the width of the inductor turn to account for the current crowding effects. For wide turns, the width of the outer mesh cells saturates to avoid underestimation of the inductor losses. The mesh along the length of the metal strips obeys a wavelength criterion. This mesh adaptation mechanism provides an optimum trade-off between accuracy and simulation time.

The inductor layout is then simulated with Momentum and the S-parameter matrix is extracted for the desired frequencies. The simulation time grows linearly with the number of frequency points. Therefore, a smart frequency sampling technique is developed in each optimization so that the necessary information is obtained with a minimum number of simulated frequency points. Section III shows some examples of smart frequency sampling.

\section{E. Stopping criteria}

Stopping criteria play an especially important role due to its impact on the computational cost. Typically, evolutionary algorithms are stopped after a predefined number of generations. However, setting this number is not a trivial problem. If the number is too low, the obtained POF will be far from the ideal one, and the usefulness of the results will dramatically decrease. If it is too high, it implies a large waste of computational resources, as each extra generation in our optimization problem may take a number of hours to compute. Our adaptive criteria aim at stopping the optimization algorithm when no significant improvement in the obtained POF is expected from allowing additional iterations. To measure such improvement, the interesting POF properties are convergence and diversity. Convergence refers to how close the obtained POF and the ideal POF are. Diversity encompasses extent (i.e., maximum range of objective values within the established constraints) and uniformity (i.e., distribution of points along the POF). As an improvement in convergence can be masked by a degradation of uniformity, monitoring three separate metrics for convergence and diversity is used here [21]. To measure uniformity, the spacing metric is used [18]. To monitor extent, a extent ratio metric must be introduced. The extent ratio is defined as the ratio of the extent metric of the populations of two consecutive generations. The extent metric is defined as the volume of the hypercube given by the minimum and maximum value of each objective in the current front. A convenient metric to monitor convergence is the generational distance (GD) defined as:

$$
G D=\frac{\left(\sum_{i=1}^{n} d_{i}^{2}\right)^{1 / 2}}{\mid \text { POF } F_{\text {approx } \mid}}
$$

where $d_{i}$ is the Euclidean distance of each member in the current front $\left(P O F_{\text {approx }}\right)$ to the closest point in the ideal POF [18]. However, the ideal POF is not known in our case. Therefore, a new metric, the dominance-based generational distance, is used [21]. The generational distance between the current and the ideal front is replaced by the generational distance between the current front and that of the previous generation, and such distance calculation is restricted to the solutions of the second front that are dominated by each solution of the first front.

\section{EXPERIMENTAL RESULTS}

This section illustrates how this methodology can be used to model the different trade-offs between inductor performances as POFs, and, at the same time, how these POFs can give very useful and powerful information to RF circuit designers or design automation tools. All experiments were performed in a $0.35-\mu m$ CMOS technology. 


\section{A. Two-Objectives Trade-offs: $L v$ s. Area and $L$ vs. $Q$}

1) Inductance vs. Area: A useful experiment to evaluate the capabilities of a technology process is to explore the inductance value that can be obtained for a given area. Therefore, a first experiment considers maximization of the inductance extrapolated at DC and minimization of inductor area, whereas the only constraint will be the maximum area allowed. A symmetric square topology with bridges at $90^{\circ}$ with differential excitation is considered. The ranges of variation of the design variables are: $1 \leq N \leq 10,10 \mu m \leq D_{I N} \leq 240 \mu m$ and $5 \mu m \leq W \leq 100 \mu m$, whereas the spacing between turns has been fixed at $2.5 \mu \mathrm{m}$, as no improvement is expected from a larger spacing. The resulting trade-off between DC inductance and area is shown in Fig.4. The grid in turn width and inner diameter is $0.05 \mu \mathrm{m}$. The grid for the number of turns, given by the layout template, is 1 . A more complex layout template would allow a grid of $1 / 4$ turn but would complicate interconnections at the circuit level.

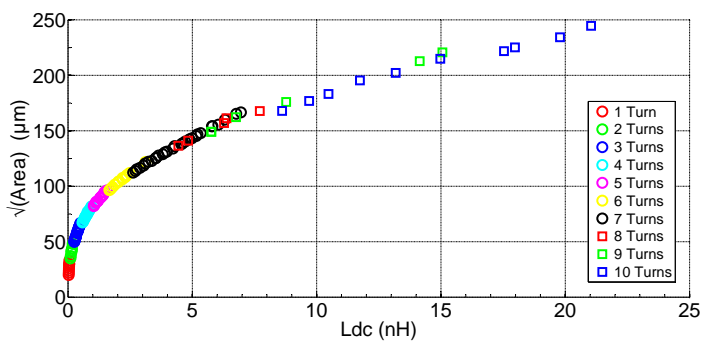

Fig. 4: Inductance vs. Area.

2) Inductance vs. Quality Factor: Another interesting trade-off to study is the achievable inductance and quality factor at a certain operating frequency within a limited area. In this case, the optimization is performed for an operating frequency, $f_{o}=2.5 \mathrm{GHz}$, whereas the inductor area is limited to $200 \mu \mathrm{m} \times 200 \mu \mathrm{m}$. The same topology of the previous experiment is used. The ranges of variation of the design variables are: $1 \leq N \leq 10,10 \mu m \leq D_{I N} \leq 190 \mu m, 5 \mu m \leq$ $W \leq 100 \mu \mathrm{m}$ and the spacing has been fixed at $2.5 \mu \mathrm{m}$. The upper bound of the inner diameter is motivated by the area limitation. Additional constraints are imposed to ensure that the inductance is sufficiently flat from DC to slightly above the operating frequency, and that the self-resonance frequency is sufficiently above this frequency. In order to keep the number of simulation points to a minimum value, a sampling strategy is planned that allows to check whether the constraints are met without simulating at a large number of frequency points. In this case, this is extremely important as determining the SRF could imply simulating a large number of frequency points for each inductor. Instead, the alternative approach in Fig. 5 is proposed. To ensure that the inductance is flat at the operating frequency, it is measured at four frequency values: $100 \mathrm{KHz}, f_{o}=2.5 \mathrm{GHz}$ and two additional frequencies, one slightly above $f_{o}$ and one slightly below $f_{o}$. Then, differences between the value of the inductance at $f_{o}$ and at $100 \mathrm{KHz}$ are constrained to be smaller than $5 \%$, and the differences between $L\left(f_{o}+\triangle f\right)$ and $L\left(f_{o}-\triangle f\right)$ must be smaller than $1 \%$. On the other hand, if we want to ensure that the SRF lies sufficiently above $f_{o}$, we have only to ensure that the value of $Q$ is near its maximum at $f_{o}$ and always with a positive slope around $f_{o}$, i.e. $Q\left(f_{o}\right)<Q\left(f_{o}+\triangle f\right)$. As it can be seen, only four frequency points are required to correctly determine the value of all the constraints. Figure 6 shows the results obtained, where inductors with 3 to 7 turns present the best $L_{e q} v s . Q$ trade-off within a given area for this technology. This tradeoff is useful, for instance, for component placement in early stages of circuit layout generation.

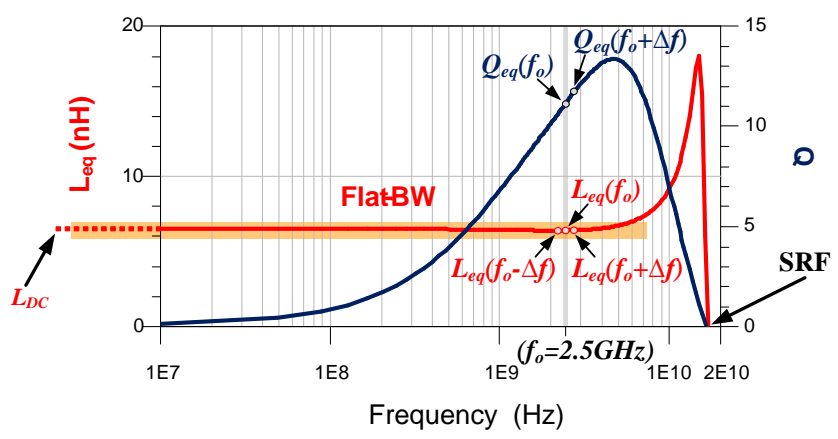

Fig. 5: Illustrating the sampling strategy.

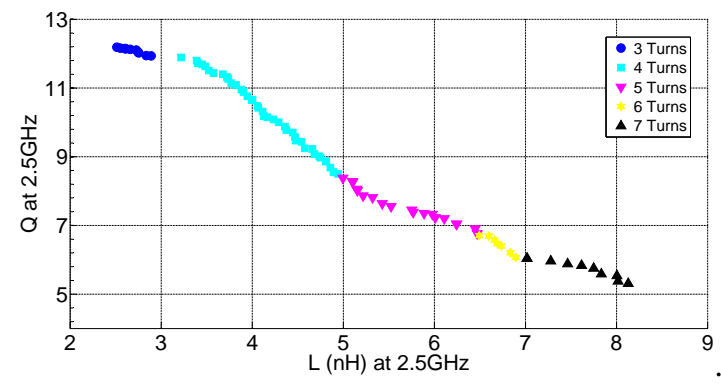

Fig. 6: POF of the Inductance vs. Quality Factor.

\section{B. Three-Objectives trade-offs: $L$ vs. $Q$ vs. Area}

The aim of this experiment is to obtain a full set of inductors that models the best trade-offs between inductance, quality factor and area, the most meaningful parameters when inductors are included in RF circuits. For these experiments, the inductance and quality factor at $f_{o}=2.5 \mathrm{GHz}$ were maximized whereas the inductor area was minimized and limited to a maximum value of $400 \mu \mathrm{m} \times 400 \mu \mathrm{m}$. The ranges of variation of the design variables are: $1 \leq N \leq 10$, $10 \mu m \leq D_{I N} \leq 390 \mu m$ and $5 \mu m \leq W \leq 100 \mu m$. Additional constraints imposed are the same described in the previous experiment.

Two different experiments are performed for two inductor topologies: symmetric square inductors with bridges at $90^{\circ}$ and spiral square inductors. The excitation mode is different for each topology: differential excitation for the former topology (symmetric) and non-differential for the latter (asymmetric). In the case of the asymmetric inductor, the fact that the input impedance is different at each port of the device due to its lack of symmetry was taken into account in the methodology. For this reason, the quality factor for each port was obtained and the best value was selected to guide the optimization flow. Results for both topologies are shown in Fig. 7. 
These results provide valuable information to circuit designers because they bring in accurate information of the price to pay if one of the inductor performances is to be improved. For example, for a given inductance we can know the possible values for the quality factor that can be obtained and the silicon area occupied by each inductor. An important fact here is that the Pareto-front is actually a library of completely sized and accurately characterized inductors with optimum performance trade-offs that can be used for circuit-level sizing. A total of 1000 symmetric inductors and 800 asymmetric inductors are obtained in these examples and the time needed to generate these fronts is about 2 weeks on a node with 2 AMD64 processors and 6 cores at $2.6 \mathrm{GHz}$.

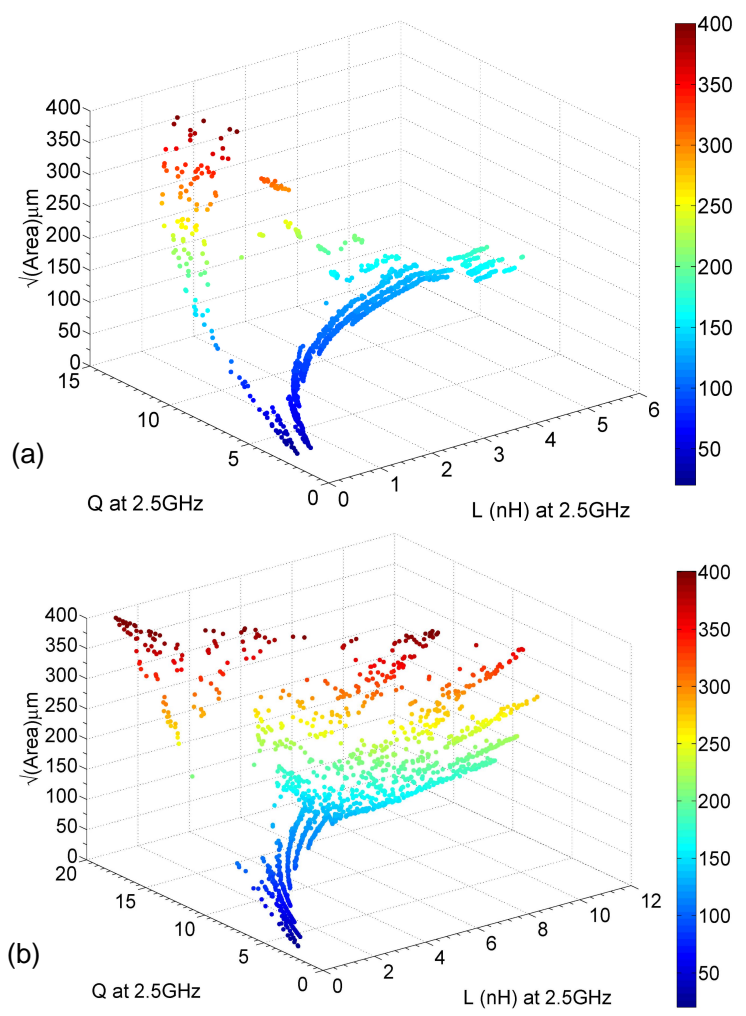

Fig. 7: Inductance vs. Quality Factor vs. Area trade-offs for (a) asymmetric square and (b) symmetric square with bridges at $90^{\circ}$ inductors.

\section{COnclusions}

A new methodology for the generation of performance fronts exhibiting the best trade-offs of integrated inductors, that provides a set of accurately simulated optimal inductors, has been presented. The generated fronts enable RF IC designers to explore the design space as well as select those inductors that better fit their requirements. Moreover, new automated RF IC design methodologies are enabled, in which Pareto optimal performance fronts of inductors are used at higher hierarchical levels, e.g., in multi-objective bottom-up design methodologies [22]. This model of use requires a highly accurate performance evaluation, which is provided by full EM simulation. The unavoidable penalty in computation time is alleviated by the offline generation in this model of use. Future work will exploit and demonstrate the use of inductor fronts for RF circuit synthesis with optimized performances.

\section{REFERENCES}

[1] M. D. M. Hershenson, A. Hajimiri, S. S. Mohan, S. P. Boyd, and T. H. Lee, "Design and optimization of LC oscillators," in IEEE/ACM Int. Conf. on Comput.-Aided Des., 1999, pp. 65-69.

[2] P. Pereira, H. Fino, and M. Ventim-Neves, "LC-VCO design methodology based on evolutionary algorithms," in Int. Conf. Synthesis, Modeling, Analysis and Simulation Methods and Applicat. to Circuit Design, Sept. 2012, pp. 189-192.

[3] A. Nieuwoudt, T. Ragheb, and Y. Massoud, "Narrow-band low-noise amplifier synthesis for high-performance system-on-chip design," $M i$ croelectronics Journal, vol. 38, no. 12, pp. 1123-1134, 2007.

[4] C. R. C. De Ranter, G. Van der Plas, M. Steyaert, G. Gielen, and W. Sansen, "CYCLONE: Automated design and layout of RF LCoscillators," IEEE Trans. Comput.-Aided Des. Integr. Circuits Syst., vol. 21, pp. 1161-1170, Oct. 2002.

[5] J. Ramos, K. Francken, G. Gielen, and M. Steyaert, "An efficient, fully parasitic-aware power amplifier design optimization tool," IEEE Trans. Circuits Syst. I, vol. 52, pp. 1526-1534, Aug. 2005.

[6] K. Choi and D. J. Allstot, "Parasitic-aware design and optimization of a CMOS RF power amplifier," IEEE Trans. Circuits Syst., vol. 53, pp. 16-25, Jan. 2006.

[7] N. A. Talwalkar, C. P. Yue, and S. S. Wong, "Analysis and synthesis of on-chip spiral inductors," IEEE Trans. Electron Devices, vol. 52, pp. 176-182, Feb. 2005.

[8] A. M. Niknejad and R. G. Meyer, "Analysis, design, and optimization of spiral inductors and transformers for Si RF IC's," IEEE J. of Solid-State Circuits, vol. 33, no. 10, pp. 1470-1481, Oct. 1998.

[9] M. D. M. Hershenson, S. S. Mohan, S. P. Boyd, and T. H. Lee, "Optimization of inductor circuits via geometric programming," in Proc. 36th ACM/IEEE Design Automation Conf., 1999, pp. 994-998.

[10] Y. Zhan and S. S. Sapatnekar, "Optimization of integrated spiral inductors using sequential quadratic programming," in Proc. Design, Automation and Test in Europe Conf., 2004, pp. 622-627.

[11] P. Pereira, H. Fino, F. Coito, and M. Ventim-Neves, "RF integrated inductor modeling and its application to optimization-based design," Anal. Integr. Circuits and Signal Process., vol. 73, no. 1, pp. 47-55, 2012.

[12] A. Nieuwoudt and Y. Massoud, "Variability-aware multilevel integrated spiral inductor synthesis," IEEE Trans. Comput.-Aided Des. Integr. Circuits Syst., vol. 25, pp. 2613-2625, Dec. 2006.

[13] S. K. Mandal, S. Sural, and A. Patra, "ANN- and PSO-based synthesis of on-chip spiral inductors for RF ICs," IEEE Trans. Comput.-Aided Des. Integr. Circuits Syst., vol. 27, pp. 188-192, Jan. 2008.

[14] W. Yu and J. W. Bandler, "Optimization of spiral inductor on silicon using space mapping," in IEEE MTT-S Int. Microw. Symp. Dig., 2006, pp. $1085-1088$

[15] M. Ballicchia and S. Orcioni, "Design and modeling of optimum quality spiral inductors with regularization and Debye approximation," IEEE Trans. Comput.-Aided Des. Integr. Circuits Syst., vol. 29, pp. 1669-1681, Nov. 2010.

[16] B. Liu, D. Zhao, P. Reynaert, and G. Gielen, "Synthesis of integrated passive components for high-frequency RF ICs based on evolutionary computation and machine learning techniques," IEEE Trans. Comput.Aided Des. Integr. Circuits Syst., vol. 30, pp. 1458-1468, Oct. 2011.

[17] S. Jeong and S. Obayashi, "Efficient global optimization (EGO) for multi-objective problem and data mining," in IEEE Congr. Evol. Comput., vol. 3, 2005, pp. 2138-2145.

[18] K. Deb, Multi objective optimization using evolutionary algorithms. John Wiley and Sons, 2001.

[19] M. Danesh and J. Long, "Differentially driven symmetric microstrip inductors," IEEE Trans. Microw. Theory Tech., vol. 50, pp. 332-341, Jan. 2002.

[20] T. Carrasco, J. Sieiro, J. M. López-Villegas, N. Vidal, R. GonzálezEchevarría, and E. Roca, "Mixed-mode impedance and reflection coefficient of two-port devices," Progress in Electromagn. Research, vol. 130, pp. 411-428, 2012.

[21] F. V. Fernández, J. Esteban-Muller, E. Roca, and R. Castro-López, "Stopping criteria in evolutionary algorithms for multi-objective performance optimization of integrated inductors," in IEEE Congr. Evol. Comput., 2010, pp. 1-8.

[22] T. Eeckelaert, T. McConaghy, and G. Gielen, "Efficient multiobjective synthesis of analog circuits using hierarchical pareto-optimal performance hypersurfaces," in Proc. Conf. on Design, Automation and Test in Europe, 2005, pp. 1070-1075. 